\title{
Comparative evaluation of rural electrification project plans: a case study in Mexico
}

\author{
D.F. Gómez-Hernández ${ }^{1}$, B. Domenech ${ }^{2,3 *}$, J. Moreira ${ }^{5}$, N. Farrera ${ }^{5}$, A. López- \\ González $^{1,2}$, L. Ferrer-Martî2,4 \\ ${ }^{1}$ Institute of Sustainability, Universitat Politècnica de Catalunya - Barcelona Tech, Spain \\ ${ }^{2}$ Institute of Industrial and Control Engineering, Universitat Politècnica de Catalunya - Barcelona Tech, Spain \\ ${ }^{3}$ Serra Hunter Fellow, Department of Management, Universitat Politècnica de Catalunya - Barcelona Tech, Spain \\ ${ }^{4}$ Department of Mechanical Engineering, Universitat Politècnica de Catalunya - Barcelona Tech, Spain \\ ${ }^{5}$ Institute of Basic and Applied Sciences, Arts and Science University of Chiapas, Mexico \\ Corresponding author $(*)$ : bruno.domenech@upc.edu; (+34) 934.016.579 \\ Av. Diagonal 647, Pavilion H, Floor 11, 08028, Barcelona, Spain
}

\begin{abstract}
Rural electrification project plans (REPP) aim to expand electricity access through adequate systems for communities without this service in developing countries. Many REPP have been developed, using different strategies in terms of energy uses covered, energy resources, electricity distribution and management models. This paper aims to analyze and compare REPP strategies. To do so, an evaluation methodology is developed, using a novel three-level assessment approach: the general definition at regional scale, the technical design at local scale, and the operation and maintenance (O\&M) management. More specifically, ad hoc criteria are defined at each level to examine REPP fulfilment through quantitative and qualitative indicators. As case study, three plans from Chiapas (Mexico) are evaluated: national grid extension with national O\&M; individual photovoltaic systems with regional O\&M; and wind-photovoltaic microgrids with local O\&M. Thus, the strengths and limitations of each REPP are identified in terms of strategies at regional scale, local scale and management model. Results show the ability of large plans to provide electricity to many families, while small plans may be adapted to the specific needs of end-users. The proposed criteria and indicators can be easily adjusted to evaluate REPP in different contexts to strengthen future plan strategies.
\end{abstract}

Keywords: rural electrification project plans; renewable energy; microgrids; isolated communities

\section{Introduction}

Access to modern energy, and electricity in particular, is essential for improving population quality of life, education and health. Nowadays, around 1.1 billion people live without access to electricity [1], mainly in rural areas of developing countries. There are several strategies to increase electricity coverage [2], such as the national grid extension, though it is often too expensive in remote, scattered and inaccessible areas [3]. Alternatively, stand-alone systems are commonly implemented, and the electricity generation and distribution must be designed [4]. In these projects, electricity generation can be based on conventional technologies such as diesel generators, which have been widely used in recent decades [5]. However, renewable energy (such as wind and solar) are increasingly receiving attention [6]. Unlike conventional technologies, renewables take advantage of local resources, reducing external dependence and can be a reliable and affordable option in rural areas of developing countries [7]. Electricity distribution can be developed by means of individual systems (independent generation and distribution for each consumption point) or microgrids (where a single generation point supplies several consumption points) [8]. Microgrids increase the wiring cost but also have significant benefits in comparison with individual systems, such as taking advantage of high resource locations or greater flexibility in consumption [9]. 
In these systems, a key point in ensuring project sustainability is the implementation of a management model to organize the technical and economic maintenance of electrification systems throughout their lifetime [10-12]. Different strategies have been used worldwide when defining management models: private, cooperative, municipal and community; each one having particular characteristics in terms of equipment ownership, end-user participation, operation and maintenance responsibilities, electricity tariffs, etc. [13]. The key difference between strategies is that management tasks can be carried out by external experts or properly trained community operators. Community management requires training inexperienced people and does not avoid the need for external experts in case of major breakdowns. However, it enables proximity and speed when solving minor and frequent technical problems, while creating local jobs [14].

The institutional framework to develop such projects are rural electrification project plans (REPP), which are designed based on social, economic and geographical elements, at governmental or institutional levels. In many countries, REPP have been implemented using different strategies in terms of the energy uses covered, the energy resources or the electricity distribution configuration $[8,15]$. For instance, a technically simple and widely utilized option is the replication of individual systems, as done in developing Asia [16], Morocco [17] and Brazil [18]. For their design, standardized demand and roughly uniform solar resources were considered. Other plans study several energy resources for electricity generation. For example, Nguyen [19] analyses the wind and solar resources in Vietnam, determining the best technology to be used at each part of the country. In order to improve supply quality, other plans develop solutions based on microgrids. Camblong et al. [20] promote wind-PV-biomass microgrids in rural Senegal, given the country's potential with these resources. Alzola et al. [21] propose a standardized electrification kit, to be replicated in many communities of Senegal. Zhang \& Kumar [22] implement a REPP in Western China to reach 8 million people using wind, PV or micro-hydro depending on the energy resources and demand. Millinger et al. [23] develop a REPP to electrify around 1,000 villages in India using PV microgrids or individual PV systems, depending on housing dispersion. Other renewable microgrid examples can be found in Bangladesh [24], Pakistan [25] or Colombia [26].

The evaluation and assessment of REPP strategies is a key issue in order to identify the strengths and weaknesses of each option, for the proper implementation of future plans. In this regard, different criteria and indicators have been developed [27,28]. From a regional perspective, Kaundinya et al. [29] compare the national electricity grid extension with off-grid systems, focusing on the institutional aspects faced by decision-makers and studying indicators such as the project lifetime cost, among others. Shyu [30] discusses the "Township Electrification Program" in China. Results show the clearly technical orientation of the plan, underestimating the ability of houses to pay for the electricity as well as overlooking end-users' and operators' training. Arraiz \& Calero [31] analyze the benefits of PV systems in Peru: mainly, more hours for productive activities and savings in traditional energy supplies (candles, batteries and lanterns). However, in the short term, the impact on incomes is not obvious. In any case, most studies examine particular situations, whose conclusions can hardly be extrapolated to other contexts $[3,32]$.

From a local perspective, more specific analyses of the communities to be electrified, according to their specific characteristics, have been carried out. Kirubi et al. [33] examine a PV microgrid in Kenya, demonstrating that access to electricity contributes to rural development and increases in productivity. Murni et al. [34] analyze two micro- 
hydro power plants in Bhutan, concluding that project success depends not only on technical aspects, but also on social factors such as the beneficiaries' participation. Leary et al. [14] analyze three wind energy electrification initiatives in rural areas of China, Nicaragua and Peru, showing the success of this technology at a local level, the suitability of microgrids for reducing costs and the generators' reliability in ensuring sustainability. Domenech et al. [7] analyze the design process of a Peruvian community, where four different technologies are used, according to the demand of the location and the energy resources available at each zone. López-González et al. [35] evaluate and compare the preliminary assessment, the design and the implementation phases of several microgridbased projects in Andean countries using different wind, PV and micro hydro electricity generation options. Finally, the sustainability of Venezuelan rural electrification projects based on diesel generators [36] and wind-PV-diesel microgrids [37] has also been examined recently in the literature.

Finally, some works also analyze project management and maintenance issues. FerrerMartí et al. [9] examine three electrification projects in the Peruvian Andes, considering their benefits in terms of new resources or services, as well as their technical and economic sustainability. Yadoo \& Cruickshank [13] study three projects in Kenya, Nepal, and Peru that use different strategies to manage electrification systems, evaluating the organization, capacity strengthening, client relationships and stakeholders' participation. Lillo et al. [38] evaluate and compare the management model of several projects using the Human Development approach, and conclude that end-users' participation strengthens project sustainability. Lillo et al. [39] evaluate the management model of a project using different technologies and energy services. Finally, López-González et al. [40] develop a comprehensive methodology to evaluate the sustainability of rural electrification programs from a management perspective.

In this context, the objective of this paper is to analyze and compare different REPP strategies aiming to extend access to electricity in isolated and low-income villages in developing countries. The evaluation encompasses an overview of the REPP strategies, rather than examining particular projects. For this purpose, a novel three-level evaluation methodology is proposed, which allows examining the key aspects faced by decisionmakers in such initiatives: (1) the general definition of the plan at regional scale, (2) the technical design at local scale considering the specific characteristics of each community, and (3) the management of the electrification systems to organize the operation and maintenance (O\&M). More specifically, ad hoc criteria are defined at each assessment level to evaluate the fulfilment of the REPP strategies through quantitative and qualitative indicators. The proposed criteria and indicators can be easily adjusted to evaluate REPP in different contexts to strengthen future plan strategies.

As case study, three plans from Chiapas (Mexico) are analyzed. The first one consists in extending the national electricity grid, the O\&M being managed by a centralized utility company. The second uses stand-alone individual PV systems and O\&M is dealt with by regional technicians. Finally, the third case implements stand-alone wind and/or PV microgrids, with the end-users being responsible for O\&M activities. The comparison of the three REPP is based on real communities electrified under each plan. Results show the ability of large plans to provide electricity access to many families, although not allowing particularization of each project; while small plans may be easily adapted to the specific need of end-users and their socioeconomic development, although at a higher cost. These results can strengthen future project planning strategies. 
The remainder of the paper is organized as follows. In Section 2, three REPP strategies implemented in Chiapas are described, which allows delimiting the scope of this research. In Section 3, the proposed methodology to evaluate the fulfilment of REPP strategies under a three-level assessment is explained, clarifying the criteria and indicators used at each level. In Section 4, the three REPP strategies from Chiapas are compared using the proposed methodology. Finally, in Section 5, the main conclusions are summarized.

\section{Rural Electrification Project Plans}

In this section, three REPP strategies implemented in Chiapas (Mexico) are described, providing a context for and delimiting the scope of this investigation. So far, very few studies focus on rural electrification with renewable energy in Mexico, mainly due to the lack of technical and economic data [41]. Gutiérrez-Vera [42] analyzes the performance of 19 technological options for the electrification of 12 remote communities, considering 11 technical and 5 socioeconomic criteria. The author focuses on a community close to the capital, recommending a wind-PV-diesel system as the best option. The same author studies another project in central Mexico [43]. A hybrid wind-PV system provides the best supply quality, operating autonomously and cost-effectively. Corral et al. [44] propose the electrification of rural villages in Northwest Mexico, using IntiGIS software to determine the most appropriate technology at each location. The authors conclude that the national grid extension is profitable for distances up to $900 \mathrm{~m}$, which represents $4 \%$ of the non-electrified rural communities in the country, while PV systems are preferable for $83 \%$ and wind power for the remaining $13 \%$.

In this context, although the electricity coverage in Mexico is relatively high, more than $10 \%$ of the houses in the Southern states have no electricity. That means around 80,000 communities, most of them with less than 100 inhabitants and without other services such as drinking water, medical assistance, educational services, etc. [45]. In terms of energy resources, Mexico has an installed capacity of around 64,045 GW [46]: 71.7\% in fossil fuels, $3.1 \%$ in nuclear energy and $25.2 \%$ in renewable energy (hydro $18.3 \%$, wind $4.1 \%$, geothermal $1.4 \%$, biomass $1.0 \%$, solar $0.3 \%$ and biogas $0.1 \%$ ). However, the country has potential for some renewable resources. For instance, the solar radiation stands out in the North of the country with a daily average above $8 \mathrm{kWh} / \mathrm{m}^{2}$ in spring and summer. The wind resource is mainly concentrated in the Tehuantepec isthmus (Oaxaca), in the South of the country, where the annual average wind speed exceeds $10 \mathrm{~m} / \mathrm{s}$ at $50 \mathrm{~m}$.a.s.l. [47].

The State of Chiapas, in the South of the country, has 4,796,580 inhabitants, with $51 \%$ living in rural villages, and has the lowest Human Development Index in Mexico (0.667). In addition, around 6,000 communities have no electricity [45]. Regarding the energy resources, the solar radiation is approximately uniform throughout the State, with a daily average of $5.7 \mathrm{kWh} / \mathrm{m}^{2}$ [48]. In fact, $42 \mathrm{~kW}$ have been installed to date in large PV projects for isolated areas. Concerning the wind resource (Figure 1), a small area in the East and, especially, the West of the State standout, as wind speeds exceed $8.5 \mathrm{~m} / \mathrm{s}$ at 50 m.a.s.l. In the following sections, three REPP developed in remote areas are described:

- Plan 1 (Section 2.1): the national grid is extended by the Mexican government, being O\&M managed by a national utility company while end-users pay for the service. This is illustrated by the project in Nuevo Amanecer Tenejapa. 
- Plan 2 (Section 2.2): stand-alone individual PV systems are installed and O\&M is regionally managed, free of charge for end-users, by the Institute for Renewable Energy of Chiapas (INER). This is illustrated by the project in Nuevo Belén.

- Plan 3 (Section 2.3): stand-alone wind and/or PV microgrids are implemented by the Arts and Science University of Chiapas (UNICACH), being O\&M managed locally by end-users. This is illustrated by the projects in El Tuzal and Villa del Río.

Figure 1-b shows the location of each community within the State of Chiapas, while the electrification projects installed are schematized in Figure 2.

\subsection{Plan 1: national electricity grid}

The national electricity grid extension is the most used strategy of the Mexican government, through the Federal Electricity Commission (CFE), for increasing electricity access. Following the current trend, this option will be utilized for most of the communities in the country. The cost of this strategy decreases with the road infrastructure quality, thereby easing access to remote areas. The goal is to reach $99 \%$ of electricity coverage throughout the country by 2018 and, for Chiapas, the electrification of 6,040 houses in 63 communities. Regarding the technical design, the grid extension is made from the nearest location, following a radial scheme. Then, the demand and the transformer to be used at each village are estimated according to standardized patterns.

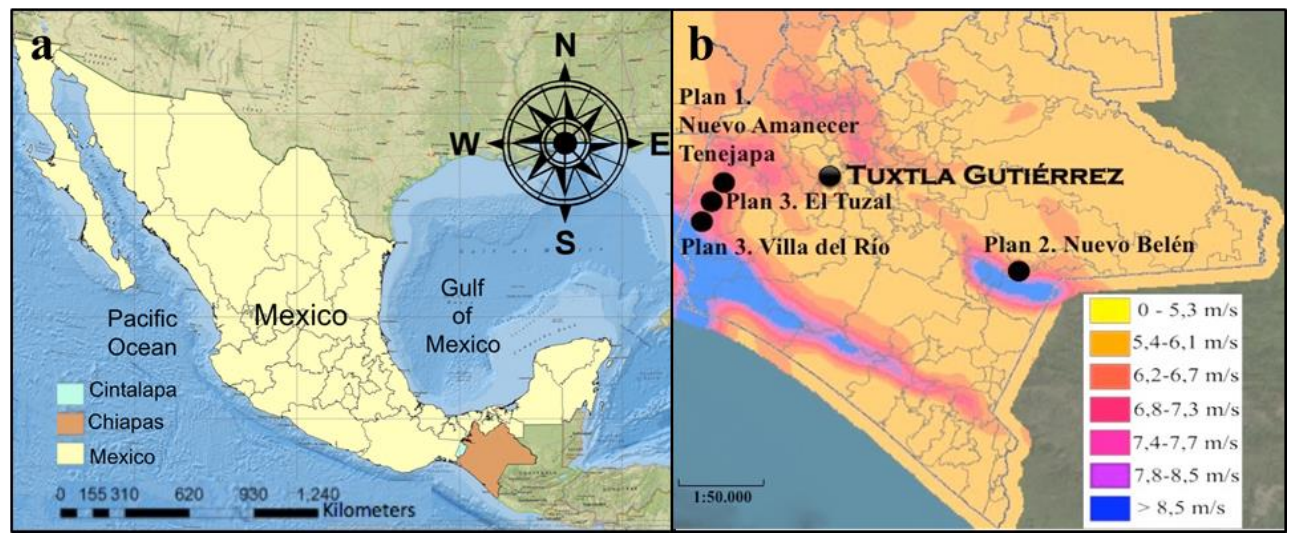

Figure 1. Location of Chiapas in Mexico (a). Wind map of Chiapas [48] and location of its capital, Tuxtla Gutiérrez, as well as the four electrification projects analyzed in this paper (b).

Nuevo Amanecer Tenejapa (Figure 2.a) is located $100 \mathrm{~km}$ from the regional capital, Tuxtla Gutiérrez, about $15 \mathrm{~km}$ from the national electricity grid, and has 81 inhabitants in 24 houses. The community also has three schools (preschool, primary and secondary) and two wells from which the inhabitants draw water. The community is located at 700 m.a.s.l. and has an average temperature of $24{ }^{\circ} \mathrm{C}$. Access to the community is not easy due to the mountainous relief and soil erosion.

In 2015, CFE extended the national electricity grid to Nuevo Amanecer Tenejapa. In particular, a medium voltage line $(13.8 \mathrm{kV})$ was used to cover the $15 \mathrm{~km}$ from the nearest location with grid access, Emiliano Zapata. Once there, a $25 \mathrm{kVA}$ transformer was installed to switch into low voltage lines $(120 \mathrm{~V})$, which were used to distribute electricity to the 24 houses, the 3 schools and 4 street lights. The low voltage grid was designed following a radial scheme. A meter was installed at each consumption point to measure consumption, so as the organization of the system is the same that CFE uses in any other 
community, town or city: meters count for the consumption of each point to establish the electricity tariff, which is usually around US\$ $1.5-2.5$ per month in poor rural areas. Families must pay this amount in the municipal capital, located $25 \mathrm{~km}$ away. In case of breakdown the end-users call CFE, who send some technicians to carry out repairs. Usually it does not take more than 1 or 2 days.

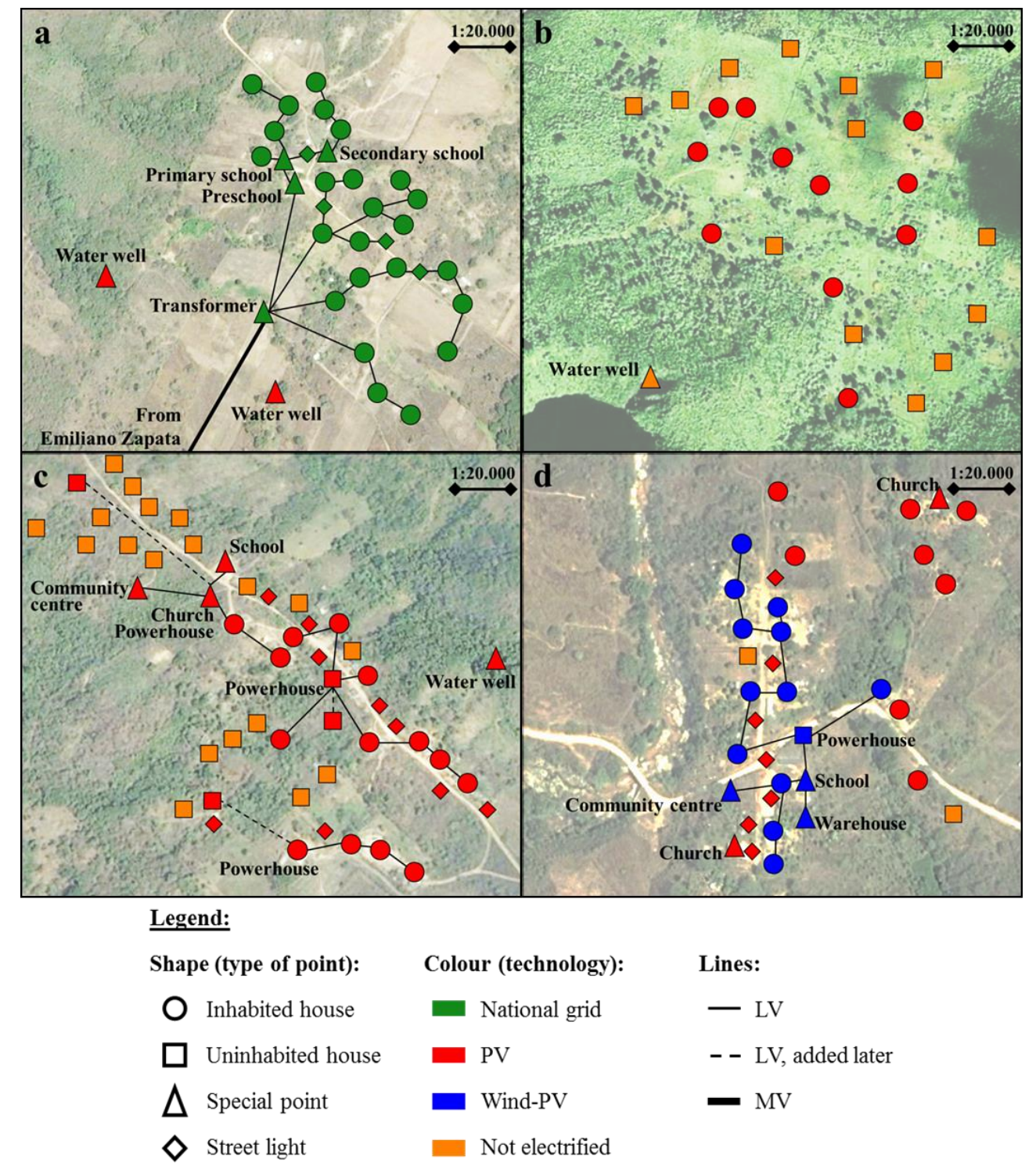

Figure 2. Map of communities studied in this paper: (a) Nuevo Amanecer Tenejapa, Plan 1; (b) Nuevo Belén, Plan 2; (c) El Tuzal, Plan 3; and (d) Villa del Río, Plan 3

\subsection{Plan 2: individual PV systems}

In 2012, CFE started the "White Flag" project plan to electrify 1,160 rural communities without electricity throughout Mexico. Given the dispersion between them and the distance from the national electricity grid, stand-alone systems were proposed. In this context, INER, an institution belonging to the regional government of Chiapas, created the program "Rural photovoltaic electrification in municipalities with very high and high rates of social backwardness in Chiapas". The goal was to install 4,395 individual PV systems in 547 extremely poor villages. This plan considers the same demand for all consumption points, established by analogy with previously electrified communities 
nearby. The solar resource is determined from government databases. The technical design is developed by selecting the PV panel that meets the demand, considering the availability of the solar resource.

Nuevo Belén (Figure 2.b) is located $200 \mathrm{~km}$ from the regional capital, Tuxtla Gutiérrez, about $4 \mathrm{~km}$ from the national electricity grid, and has 71 inhabitants in 11 houses. There are also 13 houses uninhabited. The community has neither a school nor a health center, so the population must go to nearby communities for these purposes, while the drinking water is transported from a nearby well. Nuevo Belén is located at $1500 \mathrm{~m}$.a.s.l. and has an average temperature of $22{ }^{\circ} \mathrm{C}$. The community is located $600 \mathrm{~m}$ from the main road.

The project of Nuevo Belén consists in electrifying the 11 inhabited houses with individual PV systems; each one including a $250 \mathrm{~W}$ panel, two $115 \mathrm{Ah}$ (DC, $12 \mathrm{~V}$ ) batteries to bridge the gap between generation and consumption periods, and a $450 \mathrm{~W}$ $(\mathrm{DC} / \mathrm{AC}, 120 \mathrm{~V})$ inverter to allow the use of a greater variety of appliances. The system is exactly the same as those expected to be used in other communities included in the plan, and was designed by INER technicians, taking into account a solar resource of 4.8 $\mathrm{kWh} / \mathrm{m}^{2}$ in the region and a basic consumption in lighting (light bulbs), telecommunications (TV, DVD, computer) and small appliances (blender, etc.).

Regarding O\&M activities, the head of each house is responsible for his/her own system. Consequently, they receive basic technical training: PV panel cleaning, battery charge levels, system limitations, etc. In addition, the community leader is defined as the link between INER and the population. During the design and installation, s/he is responsible for informing the inhabitants about the project's progress as well as managing small activities in which the end-users must participate. During the project's functioning, s/he is in charge of notifying INER technicians about equipment breakdowns or technical issues. Finally, it must be noted that the installation and maintenance of the systems are free for beneficiaries.

\subsection{Plan 3: wind and/or PV microgrids}

Since 2013, UNICACH has been promoting the "Network of solar communities of Chiapas (Recomsol)" plan to electrify 8 rural villages with a total population of 800 inhabitants. The plan aims to contribute to the development of such communities by taking advantage of local energy resources, and includes the electrification of houses, schools, community centers, pumping of drinking water and outdoor lighting. The plan arises from several requests, made by the communal councils of such communities, to extend the national electricity grid. Due to the high costs of this strategy, the regional government commissioned UNICACH to size and install the off-grid projects. UNICACH technicians are also responsible for the maintenance activities during the first months of the project, while the beneficiaries are expected to carry out these tasks afterwards, receiving adequate training and being supported by technicians hired by the Chiapas government to perform major repairs.

El Tuzal (Figure 2.c) is located $90 \mathrm{~km}$ from the regional capital, Tuxtla Gutiérrez, about $14 \mathrm{~km}$ from the national electricity grid, and has 49 inhabitants in 14 houses. The community has a school, a community center and a church. The houses do not have drinking water, which has to be transported from a nearby well. El Tuzal is located at an 
altitude of 600 m.a.s.l. and has an average temperature of $24^{\circ} \mathrm{C}$. The community is very difficult to access due to the mountainous relief and soil erosion.

In 2015 the electrification project was inaugurated, consisting of three microgrids, each one fed by ten $250 \mathrm{~W}$ PV panels, four $420 \mathrm{Ah}$ (DC, $24 \mathrm{~V}$ ) batteries and a $3.5 \mathrm{~kW}$ (AC / DC, $120 \mathrm{~V}$ ) inverter. The first microgrid (North) feeds the school, the community center, the church and 2 houses. The panels were installed close to the church, which is used as a powerhouse to store batteries and the inverter. The second microgrid (Center) feeds 8 houses and a powerhouse was built close to the panels' location. The third microgrid (South) feeds 4 houses, one of them being used as a powerhouse. The project also includes four $250 \mathrm{~W}$ PV panels for pumping water from the well and 9 street lights, each supplied by a $250 \mathrm{~W}$ PV panel and a 115 Ah battery.

It should be noted that, in recent years, many families have migrated, given the lack of basic services and socioeconomic opportunities in the community, as evinced by the 22 currently uninhabited houses. However, with the benefits of electricity, some of them are expected to return to the community. For that reason, the three microgrids were initially over-sized and unbalanced in terms of supplied loads, although they are expected to be progressively adjusted with the arrival of new families. In fact, three of the uninhabited houses have already been occupied, each one added to a different microgrid.

For the maintenance, repairs and replacement of equipment, each family pays US\$ 1 per month, regardless of the consumption, which is gathered in a common fund managed by the community leader. This amount was established by the population itself, according to their payment capacity. In addition, end-users participated actively during the installation process and contributed with some materials; these have been quantified, establishing a cost of US\$ 55 (in addition to the materials needed for connection) for new families wishing to be added to a microgrid. UNICACH has the sole responsibility for giving technical advice when needed and, in some cases, for carrying out repairs. In parallel, two inhabitants have been trained for the preventive maintenance of equipment.

On its behalf, Villa del Río (Figure 2.d) is located $100 \mathrm{~km}$ from the regional capital, Tuxtla Gutiérrez, about $7 \mathrm{~km}$ from the national electricity grid, and some $14 \mathrm{~km}$ from El Tuzal, with 70 inhabitants in 20 houses. The community has a school, a community center, a warehouse and two churches. Villa del Río is located at 700 m.a.s.l. and has an average temperature of $24{ }^{\circ} \mathrm{C}$. Access to the community is complicated, given the mountainous relief in the surroundings and the dirt road available.

The electrification project is significantly different from El Tuzal. On the one hand, 8 out of the 20 houses and the 2 churches already had individual PV systems when the electrification project was launched, so were not included. On the other hand, the wind resource is significant, especially in the South of the community. Therefore, the wind potential was used in combination with the solar resource, which is more constant despite having a lower potential. In particular, a single microgrid was installed, supplied by one $4.5 \mathrm{~kW}$ and one $1 \mathrm{~kW}$ wind turbine, as well as forty $250 \mathrm{~W}$ PV panels to supply 12 houses, the school, the community center and the warehouse. A powerhouse was built close to the generators to shelter the fifty-six $390 \mathrm{Ah}(\mathrm{DC}, 48 \mathrm{~V}$ ) batteries and the two $3 \mathrm{~kW}$ $(\mathrm{AC} / \mathrm{DC}, 120 \mathrm{~V})$ inverters. The project was completed with 7 street lights, each fed by a $250 \mathrm{~W}$ PV panel and two $115 \mathrm{Ah}(\mathrm{DC}, 12 \mathrm{~V})$ batteries. 
As in El Tuzal, the project was oversized, given the expected addition of new families or those with individual PV systems who had initially not been included. However, in this case the project beneficiaries decided not to accept new families as they did not want to have a lower energy availability per house. Finally, the maintenance was organized similarly to El Tuzal, each family paying US\$ 1.5 per month for the electricity and two technicians being trained for maintenance, repairs and replacements.

\section{Evaluation Methodology to Compare Rural Electrification Project Plans}

As explained before, this paper aims to evaluate and compared REPP strategies in developing countries. For this purpose, a three-level assessment methodology is developed, focusing on the key issues addressed by decision-makers in such initiatives: (1) the plan definition at regional scale, (2) the technical design at local scale, and (3) the management model. In particular, ad hoc criteria are defined at each level to evaluate the fulfilment of REPP strategies through quantitative and qualitative indicators. The definition of criteria and indicators is based on expert consultations and the literature review $[9,13,27,28,38,39]$. Figure 3 , shows these criteria groups which are closely related in an integral framework and were analyzed separately for these assessments.

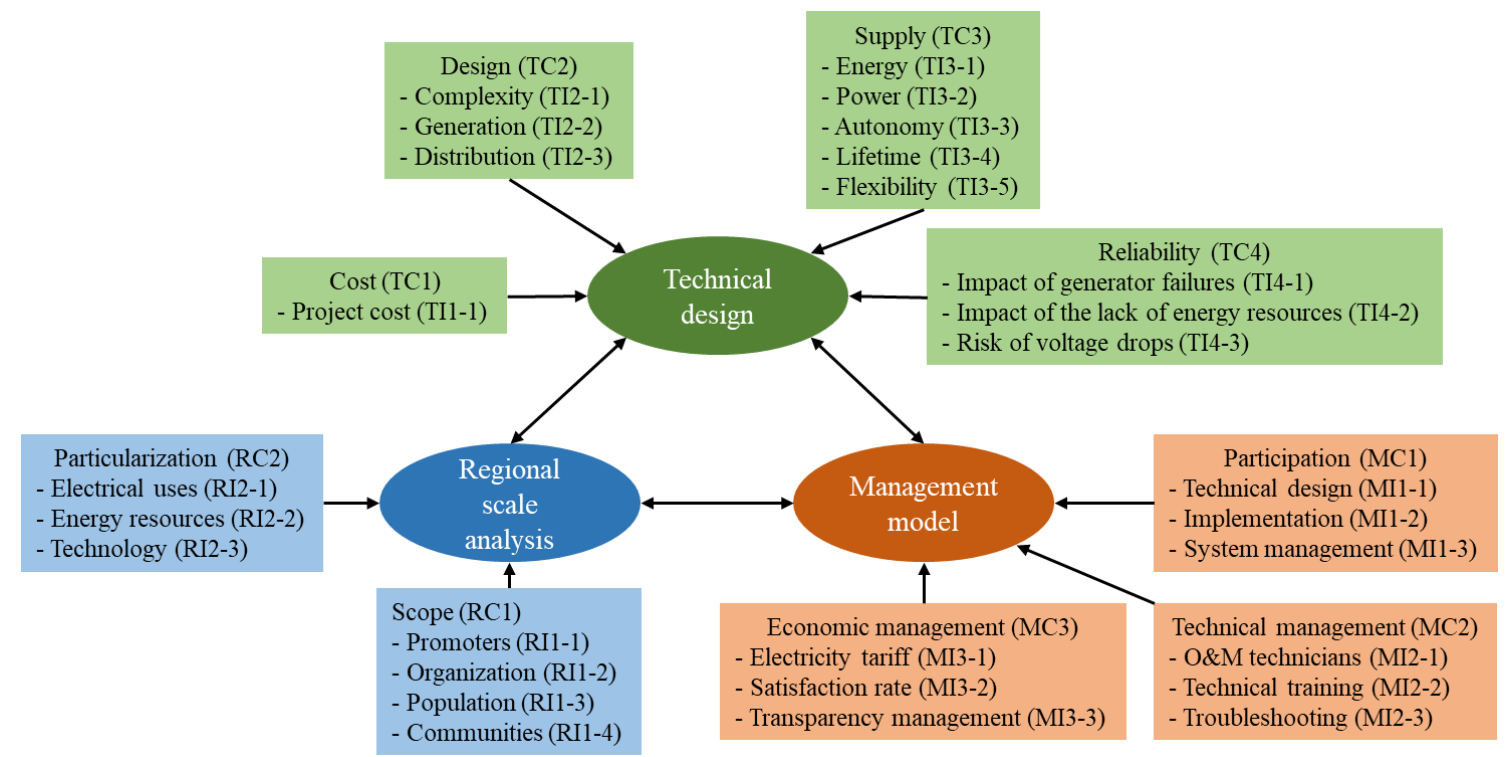

Figure 3. Scheme of criteria and indicators used in the REPP evaluation methodology

\subsection{Regional Scale Analysis}

In the regional analysis, REPP are studied from a global perspective, analyzing their scope and the particularization of the study.

- RC1, Scope: plan extension in terms of the funding institutions and the number of projects and beneficiaries reached.

o RI1-1, Promoters: scale of funding institutions: local, regional or national. Generally, the larger the scale, the greater the economic capacity, but the specific needs of each project may lose importance.

- RI1-2, Organization: type of funding institutions: public (governments, universities, etc.) or social (NGOs, universities, etc.). Public bodies tend to prioritize the benefits to the population, but can be subject to political changes. 
Social organizations pursue the benefit of the population, but may lack of economic resources.

- RI1-3, Population: estimation of the number of beneficiaries, which determines the resources needed (materials and economic).

- RI1-4, Communities: estimation of the number of benefited communities. For the same number of beneficiaries, logistics can be significantly different depending on whether they are distributed among many or few communities.

- RC2, Particularization: the degree of detail considered in the design of each plan.

○ RI2-1, Electrical uses: the applications with electricity. They can include domestic (lighting, communications, etc.), community (schools, health centers, street lights, etc.) and/or productive (productive activities, handicrafts, etc.) usages.

- RI2-2, Energy resources: the primary sources considered in the electricity supply analysis, regardless of whether they are finally used in projects of the plan or not. It may include renewable resources (solar and wind) or the national grid extension.

O RI2-3, Technology: it reflects whether the design of the electrification system in each community of the plan is replicated from the same pattern (standardized) or adapted to the specific conditions of each project (particularized).

\subsection{Technical Design}

The design of each rural electrification project is studied at local scale, considering the specific characteristics of each community and its population.

- TC1, Cost: the investment required for the project.

○ TI1-1, Project cost: it includes transportation and installation, as well as derived activities such as training for end-users. The cost is considered per family in order to standardize project comparisons and is classified as high, medium or low.

- TC2, Design: the complexity of the generation and distribution technologies used.

- TI2-1, Complexity: the estimation of the difficulty of designing the electrification project. Renewable technologies need from evaluations to estimate the variability of resources, while the national grid extension does not require it. Hybrid systems are more complicated than single technology solutions, as a balance must be found between the generation technologies. Finally, microgrids are more complex than individual systems as their structure must be designed. Considering these aspects, this indicator qualitatively defines the complexity as high, medium or low.

- TI2-2, Generation: the electricity generation technology used: wind, solar, hybrid or national grid. This indicator focuses on the technology of the electrification project; while the Energy resources (RI2-2) includes all the resources available in the community, regardless of whether they are finally used or not.

- TI2-3, Distribution: the configuration used to supply consumption points within the community: individual or microgrid; indicating the quantity of each option.

- TC3, Supply: the power supply at full system performance, the expected project lifetime and the ability to manage increases in demand over time.

- TI3-1, Energy: the energy that can be supplied per family (Wh/day), calculated from the installed generator capacity and the availability of energy resources.

- TI3-2, Power: the peak power that can be supplied per family (W), calculated from the installed inverters or transformers capacity. 
○ TI3-3, Autonomy: the self-sufficiency period in which the system can still supply the demand in the absence of energy resources, calculated from the installed battery capacity.

- TI3-4, Lifetime: the expected functioning time of the project (years).

- TI3-5, Flexibility: the ability to deal with increases in demand, either on special occasions or due to the addition of new families. This indicator qualitatively defines whether the flexibility is high, medium or low.

- TC4, Reliability: security against system limitations due to generator failures, the lack of energy resources or excessive voltage drops.

○ TI4-1, Impact of generator failures: for points depending on a single generator (or transformer), damage to such devices completely interrupts the supply. In the case of several generators, the supply still continues, although at a reduced amount.

○ TI4-2, Impact of the lack of energy resources: for points depending on a single technology, this lack completely interrupts the supply.

- TI4-3, Risk of voltage drops: the greater the proximity between generation and consumption, the lower the voltage drops, and the lower the risk of a low quality supply that could damage connected equipment.

\subsection{Management Model}

The management model examines how the population participates in and is organized around the electrification project.

- MC1, Participation: beneficiaries' involvement in the different project phases.

○ MI1-1, Technical design: the participation of beneficiaries in the electrification systems design. Including the opinion of the beneficiaries and adapting the design accordingly is important for being able to respond to their real expectations, as well as for committing them to adequately maintain the equipment. This indicator qualitatively defines whether the participation is high, medium, low or null.

○ MI1-2, Implementation: the participation of beneficiaries during the equipment installation, by providing either workforce or materials. This indicator qualitatively defines whether the participation is high, medium, low or null.

- MI1-3, System management: participation of beneficiaries in the electrification system management throughout the project's lifetime. This indicator qualitatively defines whether the participation is high, medium, low or null.

- MC2, Technical management: beneficiaries' organization for technical maintenance.

- MI2-1, O\&M technicians: the analysis of the technicians in charge of preventive maintenance tasks as well as equipment repairs. Generally, external experts have a greater technical knowledge in solving major breakdowns, while local teams promote community development and empowerment.

- MI2-2, Technical training: the end-users are generally trained in the appropriate maintenance of equipment. This training can be at a community level (complex common facilities), at residential level (basic domestic facilities) or nonexistent.

- MI2-3, Troubleshooting: evaluating the speed with which failures are managed since they occur until they are repaired, either by local or external technicians. The indicator qualitatively assesses whether troubleshooting is slow or fast. 
- MC3, Economic management: beneficiaries' organization for economic maintenance.

○ MI3-1, Electricity tariff: the amount that end-users pay for the electricity, in order to cover O\&M costs throughout the project lifetime. This rate can be free, periodic or according to consumption.

○ MI3-2, Satisfaction rate: opinion of the electricity tariff. A negative perception can cause problems among neighbors, the delay of payments, or project abandonment. This indicator qualitatively evaluates whether the satisfaction is high, medium or low.

- MI3-3, Transparency management: clarity when managing the funds raised from the electricity tariff. This feature can be the origin of conflicts among neighbors, in cases of poor management or limited economic resources. This indicator evaluates transparency as high, medium or low, also indicating the responsible.

\section{Comparative Evaluation of Rural Electrification Project Plans}

Once the standardized criteria and indicators have been defined (Section 3), the evaluation methodology can be used to assess the three REPP strategies developed in Chiapas (Section 2). The objective is to identify the strengths and limitations of each plan, in terms of the decision-making processes followed at the regional scale analysis, the technical design and the management model. To do so, each plan is carefully examined, gathering data from different sources of information:

- Regarding regional scale indicators, national, regional and local government databases including general socioeconomic data about the communities and its population are consulted. This information is confirmed with the REPP developers, who know the communities as well as the global process of each plan.

- Concerning local scale indicators, the technical reports made by the REPP developers before, during and after projects implementation are examined. Such documents include details about socioeconomic aspects of each community as well as technical design issues and the demand requirements. In addition, the responsible technicians are interviewed about aspects related to reliability of electrification systems.

- Regarding management indicators, regular project visits are made, particularly after projects implementation. Thus, the performance and organization of projects are monitored. In addition, semi-structured interviews are carried out to end-users about techno-economic issues of electrification systems and their perception. To do so, a representative sample of beneficiaries is selected, including community leaders and representatives of all social groups (children, women, elders, etc.), in order to gather the opinion of everybody and avoid biased results. This information is complemented by interviews to the REPP developers and the technicians in charge of O\&M activities.

Table 1 summarizes the information compiled about the 4 projects studied in this paper, which is discussed in this section. For Plan 3, the regional analysis is shown jointly for the two projects described, while the local scale technical design and the management model are presented separately, since the decisions taken were not always the same. 
Table 1 - Criteria and indicators for the 3 rural electrification plans studied

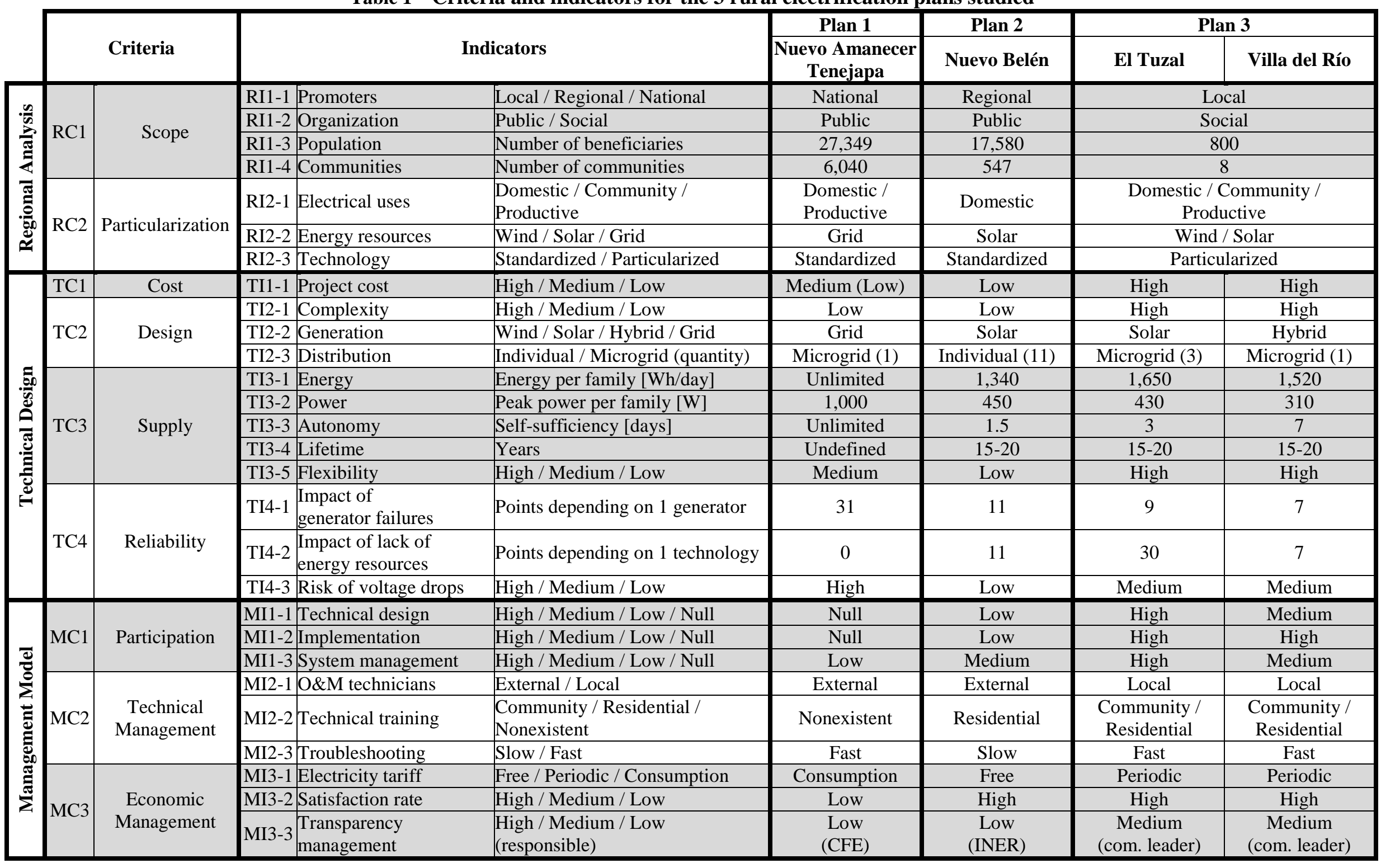




\subsection{Regional Scale Analysis}

In terms of the REPP scope (RC1), the three plans are promoted by institutions from different levels (RI1-1): national in Plan 1, regional in Plan 2 and local in Plan 3. This is related to the number of population (RI1-3) and communities (RI1-4) benefited, which are significantly higher in larger projects. Thus, Plan 1 benefits more than 27,000 people and 6,000 communities, while Plan 3 only reaches around 800 people and 8 communities.

In addition, the scale of promoters (RI1-1) and the number of population (RI1-3) and communities (RI1-4) benefited limit the ability to particularize the analysis for each project (RC2). The technical solutions used (RI2-3) in larger REPP tend towards standardization (Plan 1 and 2), without taking into account alternatives based on the most adequate energy resources (RI2-2). In Plan 1, only the national electricity grid extension is considered; while in Plan 2, the replication of the same scheme is proposed at all consumption points: individual PV systems. This last strategy has been widely used in remote areas worldwide given its technical simplicity and adaptability, since the sun has a suitable potential for electricity generation in many areas. In contrast, in the smallest plan (Plan 3), the most appropriate technical solution is studied in detail for each community. This is the only REPP where more than a single generation technology is analyzed (wind, solar or a combination of both). An individualized solution is proposed for each case, based on an examination of the electrical needs of the population and the availability of energy resources. This is reflected, as mentioned later, by a higher design complexity (TI2-1) for both the generation (TI2-2) and distribution (TI2-3) technologies.

The ability to particularize the study for each project is also related to the detailed analysis of demand in order to ensure that the electrical uses covered (RI2-1) are enough to meet the population's real needs. Plan 2, with high standardization, is limited to the electrification of houses and does not include, for example, water pumping at the community well of Nuevo Belén, which would definitely improve the living conditions of the population. In contrast, Plan 3, much more particularized, includes electrification of community spaces to meet specific needs and promotes the comprehensive socioeconomic development of the population. It should be also noted that particularization and the greater variety of electrical uses covered are promoted especially by social institutions (RI1-2), as in Plan 3, where the beneficiaries become particularly relevant in ensuring the project's success. Indeed, for social institutions, an adequate monitoring of electrification projects in the medium and long term is harder than for public institutions, such as national or regional governments. This is directly related to the beneficiaries' participation (MC1), as highlighted below.

Finally, differences in the target of villages for each plan, in terms of community density of houses and distance to the closest urban center with electricity, must be highlighted (Figure 4). The national grid extension allows the larger number of houses to be electrified, mainly thanks to its economic feasibility in communities with high density of houses, usually located closer to urban centers than more scattered villages. Rural microgrids have been implemented in settlements having a significant number of houses concentrated close to each other to avoid higher voltage drops than permitted premises. Finally, individual PV systems are installed in dispersed houses, so as microgrids would have significant voltage drops. In other words, from a regional perspective, the fastest electrification option is grid extension, followed by isolated microgrids and individual systems. This conclusion is aligned with other works from the literature $[49,50]$. 


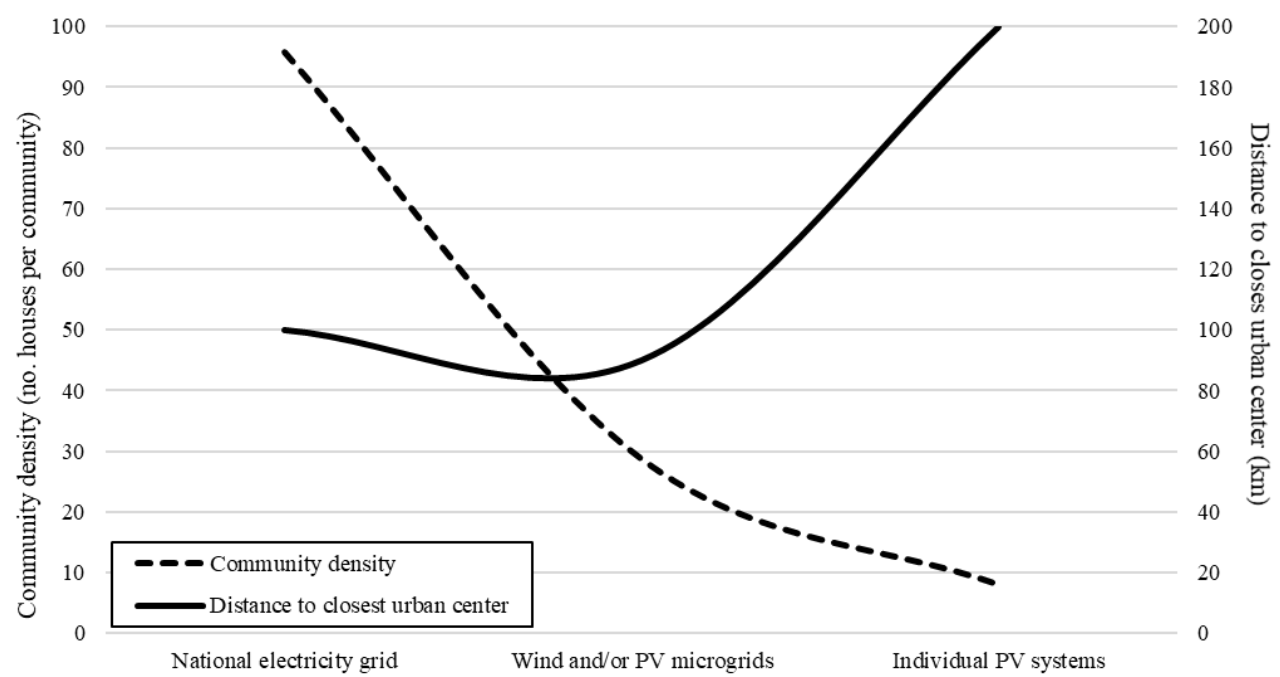

Figure 4. REPP comparison considering community density and distance to closest urban center

\subsection{Technical Design}

Project costs (TC1, TI1-1) are significantly different for each REPP. In Plan 3, the costs are higher than in Plan 2, with a low cost, or Plan 1, with a medium cost. It should be noted that in Plan 1 the cost for Nuevo Belén is medium, since the community is located $15 \mathrm{~km}$ from the national grid, a noteworthy distance that increases line extension costs. Nevertheless, for other projects included in that plan, the cost could be significantly lower. In any case, the lower cost for Plans 1 and 2 is directly related to the easier project design (TC2, TI2-1), in terms of the generation (TI2-2) and distribution (TI2-3) technologies used. Both plans replicate technically simple solutions: the extension of the national grid or individual PV systems at all consumption points. Therefore, standardization (RI2-3) is facilitated and costs can be reduced due to economies of scale, both in equipment and technicians in charge of the system's design and installation. In contrast, a much more detailed analysis of demand and energy resources is performed in Plan 3, adapting the technical design accordingly; for example, using more or fewer microgrids depending on the end-users' location or installing generators, according to the availability of the wind and solar resources.

In terms of electricity supply (TC3), the national grid extension, Plan 1, offers the best quality (TI3-1, TI3-2, TI3-3, TI3-4). The only limitation is the cost of electricity that the beneficiaries face according to their consumption (MI3-1), so most families show a low satisfaction (MI3-2) given their limited incomes. For their part, individual PV panels, Plan 2, offer the most limited electricity service, though free of any cost for end-users. Finally, Plan 3 projects, although more expensive (IT1-1), have a greater flexibility regarding consumption, being able to face demand increases (TI3-5), and have an improved quality of supply, thus promoting socioeconomic community development.

Although Plan 1 offers a better electricity supply (TC3), some limitations can be observed in terms of reliability (TC4). Indeed, in the case of a transformer failure, the whole community is harmed (TI4-1) as the supply to all consumption points depends on this device. In addition, as the village of Nuevo Belén is connected to an endpoint of the national grid, there may be significant voltage drops that can damage connected equipment (TI4-3). In contrast, the main risk for Plan 2 lies in the generator's reliability, since each consumption point depends on its single PV panel, or the lack of solar 
resources, as battery autonomy is not very high. Finally, the reliability of Plan 3 is adequate when facing breakdowns (TI4-1): the only points depending on a single generator are the street lights. Similarly, reliability is also good when faced with a lack of energy resources, since battery autonomy (TI3-3) is larger than the expected periods without wind or sun; and it is especially good for Villa del Río given the combination of generation technologies (TI4-2). In addition, microgrid voltage drops are not very significant since the distances between connected points are not long.

Figure 5 shows a visual comparison between the four studied projects for the four criteria belonging to the technical design assessment. This graphical representation has been obtained by, first, transforming the indicator evaluations into a 0 to 1 scale, according to the lower or higher performance of a project in front of the others, respectively. Then, the average value for each criterion is calculated and represented. As observed, the projects from Plan 3 get a significantly lower performance in terms of the cost (TC1) and design (TC2) than the projects from Plans 1 and 2. In contrast, they achieve a slightly higher reliability (TC4) than Plans 1 and 2; and a higher supply quality (TC3) than Plan 2, while Plan 1 takes advantage from a high supply source as the national grid.

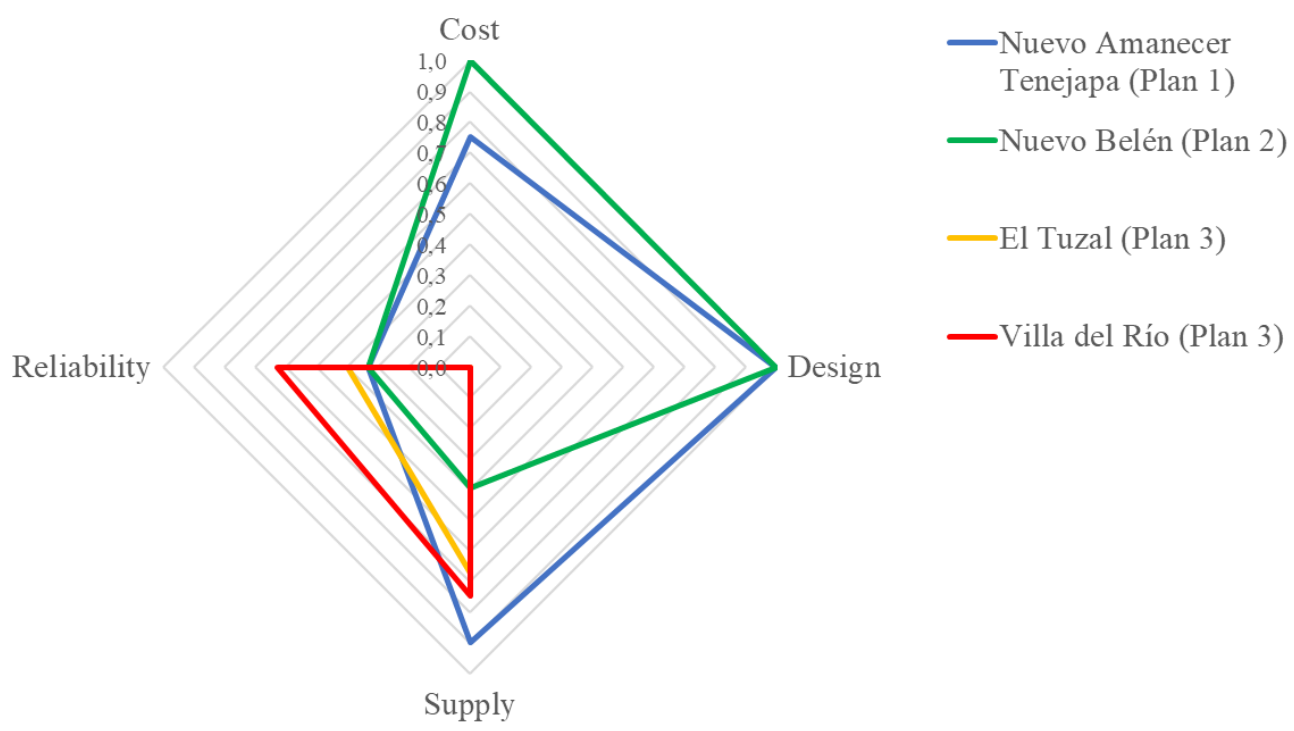

Figure 5. REPP comparison considering the criteria related to the technical design assessment

\subsection{Management Model}

There are key differences between the REPP strategies implemented. On the one hand, increasing the scope of electrification plans (RC1) complicates the participation of the beneficiary population (MC1). In large Plans 1 and 2, the attention given to end-users during project design, implementation and management is limited (MI1-1, MI1-2, MI13). In Plan 2, there is a slightly higher participation, since the community was the pilot project. On the other hand, in the smaller sized Plan 3, end-users have a greater participation in the whole project process. Participation is also encouraged when the promoters are social entities (RI1-2), to ensure projects' medium and long term sustainability.

With regards to the technical management (MC2), in large scope Plans 1 and 2 (RC1) the O\&M technicians (MI2-1) are external to the community. This scheme allows maintenance costs to be reduced, concentrating efforts in a single institution for several 
communities, while ensuring the quality of repairs made by specialized experts. However, the speed of repairs (MI2-3) is still an issue in the second REPP, probably because the plan has only recently been launched. In contrast, in Plan 3, two technicians from the communities themselves are adequately trained and responsible for such activities. This process aims to strengthen population development by not only providing access to electricity, but creating local jobs, knowledge and motivation to be self-sufficient. However, a certain risk exists in the medium and long term when dealing with major repairs of equipment, so the regional government has hired expert technicians to support the inhabitants in such events. In addition, as projects included in Plans 2 and 3 are isolated and autonomous, end-users are trained to perform basic equipment maintenance (MI2-2) at a residential level, and in Plan 3, for an efficient and productive use of electricity to improve socioeconomic development. In contrast, in Plan 1 the community is added to the national electricity grid and the existing management structures.

Finally, regarding the economic management (MC3), each plan opts for a different tariff to cover the costs of electricity (MI1-1). In Plan 1, this value depends on the consumption, as for any user included within the national grid. The tariff covers CFE expenses for the grid management and maintenance. However, in rural areas with low incomes, payments can be proportionally higher, limiting end-users' satisfaction (MI1-2). In Plan 2, a free service is chosen, subsidized by the regional government through INER. Logically, endusers' satisfaction is high, but there is a risk of misappropriation and poor maintenance of equipment, which could lead to higher repair costs. Finally, in Plan 3 each community chooses its own self-management scheme, and the promoting institutions are not involved in this matter. Although the initial idea was to provide free access to electricity, both communities opted for a monthly payment per family (regardless of consumption) in order to face maintenance, repair and substitution tasks. These funds are managed by the community leaders together with other revenues, such as the amount paid by newly connected families. In both cases, the population is satisfied with their decision (MI1-3) and can easily access and request information on the economic management (MI3-3).

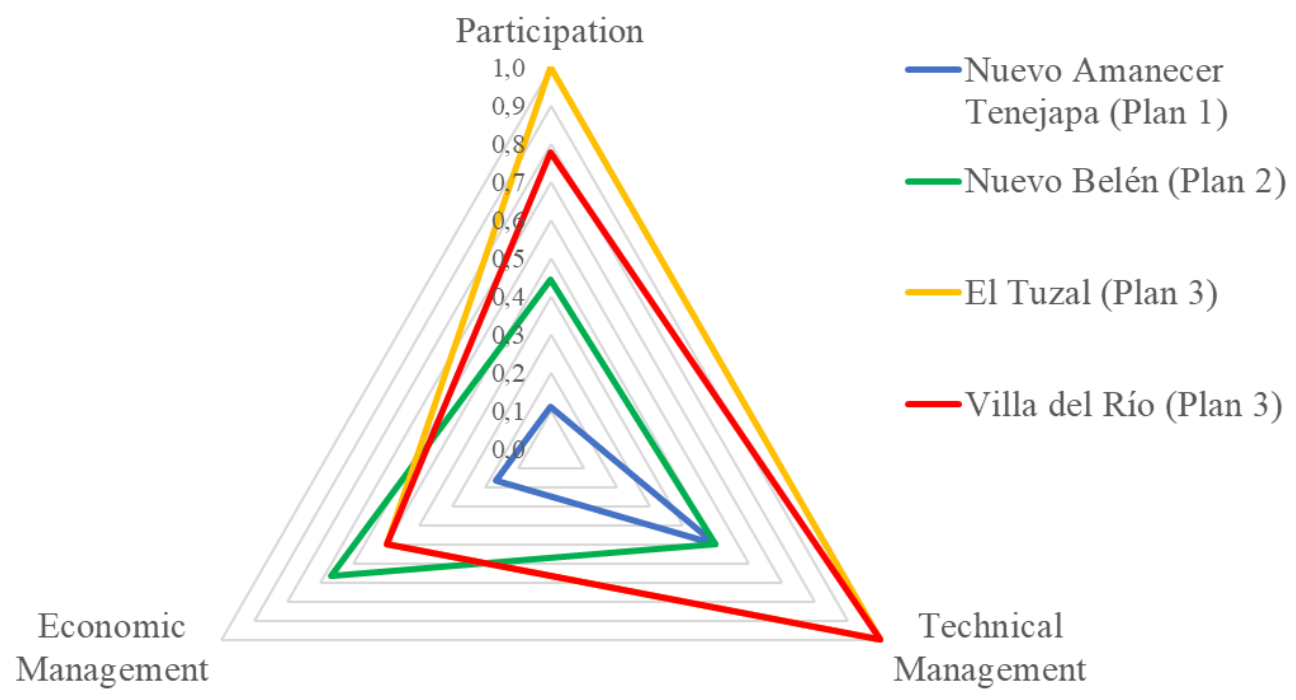

Figure 6. REPP comparison considering the criteria related to the management model assessment

Figure 6 shows a visual comparison between the four studied projects for the three criteria belonging to the management model assessment. This graphical representation has been obtained in a similar way than for the technical design. As observed, the projects from Plan 3 achieve a significantly higher performance in terms of the participation (MC1) and 
technical management (MC2) than the projects from Plans 1 and 2. In contrast, the free electricity service in Plan 2 makes the project more robust for the economic management (MC3), while Plan 1 obtains the lower performance, not being adapted to the real socioeconomic conditions of rural population.

\section{Conclusion and Policy Implications}

Despite several initiatives worldwide, rural electrification project plans (REPP) are still a challenge in many regions of developing countries. The purpose of this paper is to analyze and compare REPP strategies in developing countries. For this purpose, an evaluation methodology is developed under a three-level assessment approach to examine the key issues addressed by decision-makers: (1) the regional scale analysis, (2) the local scale technical design, and (3) the management model. Ad hoc criteria are defined to evaluate the fulfilment of REPP strategies at each assessment level through some quantitative and qualitative indicators. Hence, a comprehensive framework is achieved, which allows a differentiated understanding of REPP strategies, identifying their main strengths and limitations. As case study, three plans developed in Chiapas (Mexico) are studied. The three REPP use diverse options to expand electricity access in remote areas, as well as for project management. The first one extends the national electricity grid, which is the option commonly utilized by central governments, and O\&M management is centralized. The second plan implements individual PV systems, a common and technically simple solution, while a regional O\&M scheme is defined. The third plan uses wind and/or PV microgrids, which are increasingly being used given their good performance, being O\&M responsibilities delegated to end-users.

Results show that the national grid extension is a large plan, where end-users adopt the role of consumers who pay for a service without any active participation. Therefore, in spite of the good service quality and the efficient maintenance management, disconformity appears because the cost of electricity is not suitable for the low incomes of rural population. The use of individual PV systems is a technically simple and relatively low cost solution that provides access to a sufficient amount of energy. The main limitation depends on the system's reliability against breakdowns and the lack of resources, as end-users depend on a single generator. Finally, in small plans, the use of wind and/or PV microgrids, together with a detailed assessment of demand and energy resources, allows the electrification project to be adapted to the specific needs of each community, thus promoting the inhabitants' comprehensive socioeconomic development. In contrast, these projects are more expensive and require the motivation of end-users to ensure their medium and long term sustainability.

In conclusion, each strategy has its advantages and limitations. On the one hand, large electrification plans can provide electricity access to many families, but they do not allow participation and particularization, which can lead to limitations on the energy uses and services. On the other hand, small electrification plans may be more complete and beneficial in terms of the electricity supply and the socioeconomic development of the inhabitants, though at a higher cost. As future research, the methodology could be adapted in order to evaluate REPP strategies in other contexts, thus strengthening future plans. In this regard, most of the proposed criteria could remain the same, since they have been developed under a wide perspective, based on expert consultations and the literature review. However, the way as the indicators are measured should be adapted or re- 
considered depending on the information available. In addition, aspects such as the environmental dimension of REPP strategies could be analyzed, particularly when assessing plans based on the diesel technology.

\section{Acknowledgements}

This study was supported by the Spanish MICINN, project ENE2015-67253-R, the Centre for Development Cooperation of the Universitat Politècnica de Catalunya - Barcelona Tech (UPC), the UCD Energy21 program, co-financed through the Marie Curie FP7-PEOPLE-2013-COFUND program, and the Serra Hunter Fellowship Program of the Generalitat de Catalunya.

\section{References}

[1] IEA. World Energy Outlook - Executive Summary. Paris: International Energy Agency; 2017.

[2] Banal-Estañol A, Calzada J, Jordana J. How to achieve full electrification: lessons from Latin America. Energy Policy 2017; 108:55-69.

[3] Ahlborg H, Hammar L. Drivers and barriers to rural electrification in Tanzania and Mozambique grid-extension, off-grid, and renewable energy technologies. Renew Energy 2014; 61:117-24.

[4] Palit D, Bandyopadhyay KR. Rural electricity access in South Asia: is grid extension the remedy? A critical review. Renew Sustain Energy Rev 2016; 60:1505-15.

[5] van Ruijven BJ, Schers J, van Vuuren DP. Model-based scenarios for rural electrification in developing countries. Energy 2012; 38(1):386-397.

[6] Bekele G, Tadesse G. Feasibility study of small hydro/PV/wind hybrid system for off-grid rural electrification in Ethiopia. Appl Energy 2012; 97:5-15.

[7] Domenech B, Ferrer-Martí L, Lillo P, Pastor R, Chiroque J. A community electrification project: combination of microgrids and household systems fed by wind, PV or micro-hydro energies according to micro-scale resource evaluation and social constraints. Energy Sustain Dev 2014; 23:275-85.

[8] Narula K, Nagai Y, Pachauri S. The role of decentralized distributed generation in achieving universal rural electrification in South Asia by 2030. Energy Policy 2012; 47:345-57.

[9] Ferrer-Martí L, Garwood A, Chiroque J, Ramirez B, Marcelo O, Garfí M, Velo E. Evaluating and comparing three community small-scale wind electrification projects. Renew Sustain Energy Rev 2012; 16(7):5379-90.

[10] Palit D. Solar energy programs for rural electrification: experiences and lessons from South Asia. Energy Sustain Dev 2013; 17(3):270-9.

[11] Shyu CW. End-users' experiences with electricity supply from stand-alone mini-grid solar PV power stations in rural areas of western China. Energy Sustain Dev 2013; 17(4):391-400.

[12] Alam M, Bhattacharyya S. Are the off-grid customers ready to pay for electricity from the decentralized renewable hybrid mini-grids? A study of willingness to pay in rural Bangladesh. Energy 2017; 139(1): 433-446.

[13] Yadoo A, Cruickshank H. The role for low carbon electrification technologies in poverty reduction and climate change strategies: a focus on renewable energy mini-grids with case studies in Nepal, Peru and Kenya. Energy Policy 2012; 42:591-602.

[14] Leary J, While A, Howell R. Locally manufactured wind power technology for sustainable rural electrification. Energy Policy 2012; 43:173-83.

[15] Slough T, Urpelainen J, Yang J. Light for all? Evaluating Brazil's rural electrification progress, 2000-2010. Energy Policy 2015; 86:315-27.

[16] Urmee T, Harries D, Schlapfer A. Issues related to rural electrification using renewable energy in developing countries of Asia and Pacific. Renew Energy 2009; 34(2):354-57.

[17] Carrasco LM, Martín-Campo FJ, Narvarte L, Ortuño MT, Vitoriano B. Design of maintenance structures for rural electrification with solar home systems. The case of the Moroccan program. Energy 2016; 117(1):47-57.

[18] van Els RH, de Souza Vianna JN, Brasil ACP. The Brazilian experience of rural electrification in the Amazon with decentralized generation - the need to change the paradigm from electrification to development. Renew Sustain Energy Rev 2012; 16(3)1450-61.

[19] Nguyen K. Alternatives to grid extension for rural electrification: decentralized renewable energy technologies in Vietnam. Energy Policy 2007; 35(4):2579-89. 
[20] Camblong H, Sarr J, Niang AT, Curea O, Alzola JA, Sylla EH, et al. Microgrids project, part 1: analysis of rural electrification with high content of renewable energy sources in Senegal. Renew Energy 2009; 34(10):2141-50.

[21] Alzola JA, Vechiu I, Camblong H, Santos M, Sall M, Sow G. Microgrids project, part 2: design of an electrification kit with high content of renewable energy sources in Senegal. Renew Energy 2009; 34(10):2151-9.

[22] Zhang X, Kumar A. Evaluating renewable energy-based rural electrification program in western China: emerging problems and possible scenarios. Renew Sustain Energy Rev 2011; 15(1):773-9.

[23] Millinger M, Marlind T, Ahlgren EO. Evaluation of Indian rural solar electrification: a case study in Chhattisgarh. Energy Sustain Dev 2012; 16(4):486-92.

[24] Bala B, Siddique SA. Optimal design of a PV-diesel hybrid system for electrification of an isolated island - Sandwip in Bangladesh using genetic algorithm. Energy Sustain Dev 2009; 13(3):137-42.

[25] Ahmad J, Imran M, Khalid A, Iqbal W, Ashraf SR, Adnan M, Ali SF, Khokhar KS. Techno economic analysis of a wind-photovoltaic-biomass hybrid renewable energy system for rural electrification: A case study of Kallar Kahar. Energy 2018 (in press, accepted manuscript); X(X):X-X.

[26] Mamaghani AH, Escandon SAA, Najafi B, Shirazi A, Rinaldi F. Techno-economic feasibility of photovoltaic, wind, diesel and hybrid electrification systems for off-grid rural electrification in Colombia. Renew Energy 2016; 97:293-305.

[27] Vera I, Langlois L. Energy indicators for sustainable development. Energy 2007; 32(6):875-882.

[28] Ilskog E, Kjellstrom B. And then they lived sustainably ever after? - Assessment of rural electrification cases by means of indicators. Energy Policy 2008; 36(7):2674-84.

[29] Kaundinya DP, Balachandra P, Ravindranath PH. Grid-connected versus stand-alone energy systems for decentralized power - a review of literature. Renew Sustain Energy Rev 2009; 13(8):2041-50.

[30] Shyu CW. Rural electrification program with renewable energy sources: an analysis of China's township electrification program. Energy Policy 2012; 51:842-53.

[31] Arraiz I, Calero C. From candles to light: the impact of rural electrification. Inter-American Development Bank, IDB working paper series no. IDB-WP-599; 2015.

[32] Wamukonya N, Davis M. Socio-economic impacts of rural electrification in Namibia: comparisons between grid, solar and unelectrified households. Energy Sustain Dev 2001; 5(3):5-13.

[33] Kirubi C, Jacobson A, Kammen DM, Mills A. Community-based electric micro-grids can contribute to rural development: evidence from Kenya. World Dev 2009; 37(7):1208-21.

[34] Murni S, Whale J, Urmee T, Davis J, Harries D. The role of micro hydro power systems in remote rural electrification: a case study in the Bawan Valley, Borneo. Proced Eng 2012; 49:189-96.

[35] López-González A, Domenech B, Gómez-Hernández D, Ferrer-Martí L. Renewable microgrid projects for autonomous small-scale electrification in Andean countries. Renewable and Sustainable Energy Reviews 2017; 79:1255-65.

[36] López-González A, Domenech B, Ferrer-Martí L. Lifetime, cost and fuel efficiency in diesel projects for rural electrification in Venezuela. Energy Policy 2018; 121: 152-61.

[37] López-González A, Domenech B, Ferrer-Martí L. Sustainability and design assessment of rural hybrid microgrids in Venezuela. Energy 2018; 121: 229-42.

[38] Lillo P, Ferrer-Martí L, Boni A, Fernández-Baldor A. Assessing management models for off-grid renewable energy electrification projects using the human development approach: case study in Peru. Energy Sustain Dev 2015; 25:17-26.

[39] Lillo P, Ferrer-Martí L, Fernández-Baldor A, Ramírez B. A new integral management model and evaluation method to enhance sustainability of renewable energy projects for energy and sanitation services. Energy Sustain Dev 2015; 29:1-12.

[40] López-González A, Domenech B, Ferrer-Martí L. Formative evaluation of sustainability in rural electrification programs from a management perspective: a case study from Venezuela. Renewable and Sustainable Energy Reviews 2018; 95:95-109.

[41] SENER. Renewable energy prospect 2012-2026. Mexico DF: National Secretary for Renewable Energy; 2012.

[42] Gutiérrez-Vera J. Options for rural electrification in Mexico. IEEE Trans Energy Convers 1992; 7(3):426-33.

[43] Gutiérrez-Vera J. Use of renewable sources of energy in Mexico. Case: San Antonio Agua Bendita. IEEE Trans Energy Convers 1994; 9(3):442-50.

[44] Corral BA, Domínguez J, Serrano M. Analysis of technological competitiveness for rural electrification in the municipality of Ensenada, Mexico. Madrid: Technical Report of the Research Centre for Energy - Environment and Technology (CIEMAT) - Spanish Ministry of Science and Education; 2013. 
[45] INEGI. National census of population and housing. Mexico DF: National Institute of Statistics and Geography; http://www.beta.inegi.org.mx/, 2010 [accessed 10.12.2017].

[46] SENER. Clean energy progress report 2015. Mexico DF: National Secretary for Renewable Energy; 2015.

[47] IRENA. Renewable energy prospects: Mexico - REmap 2030 analysis. Abu Dhabi: International Renewable Energy Agency - Mexican Secretary of Energy; 2015.

[48] IIE. Geographical information system for renewable energy in Mexico. Mexico DF: Institute of Electrical Research; http://sag01.iie.org.mx/siger, 2016 [accessed 18.12.2017].

[49] Dugoua E, Liu R, Urpelainen J. Geographic and socio-economic barriers to rural electrification: new evidence from Indian villages. Energy Policy 2017; 106:278-87.

[50] Zvoleff A, Kocaman AS, Huh WT, Modi V. The impact of geography on energy infrastructure costs. Energy Policy 2009; 37:4066-78. 